\title{
Force/Position Hybrid Control of 6PUS-UPU Redundant Driven Parallel Manipulator Based on 2-DOF Internal Model Control
}

\author{
Kuijing Zheng and Chao Wang \\ Key Laboratory of Advanced Forging \& Stamping Technology and Science and Hebei Provincial Key Laboratory of \\ Parallel Robot and Mechatronic System, Yanshan University, Qinhuangdao 066004, China
}

Correspondence should be addressed to Kuijing Zheng; kjzheng@ysu.edu.cn

Received 16 September 2014; Accepted 16 November 2014; Published 8 December 2014

Academic Editor: Hung-Yuan Chung

Copyright (C) $2014 \mathrm{~K}$. Zheng and C. Wang. This is an open access article distributed under the Creative Commons Attribution License, which permits unrestricted use, distribution, and reproduction in any medium, provided the original work is properly cited.

\begin{abstract}
To improve control performance of parallel manipulator, servo control is optimized according to 2-DOF control with Internal Model Control. Position loop of the controller is redesigned based on the original current loop and speed loop. The hybrid force/position control strategy on the basis of cross-coupling is proposed. With mechatronic cosimulation system, it is proved that the force/position hybrid control on the basis of 2-DOF Internal Model Control has better stability and position precision compared with the traditional PID control. The control precision and stability of the parallel manipulator are improved effectively in actual experiment. Two types of compliance operation including peg in hole and surface tracking are realized in 6PUS-UPU parallel manipulator and both have good applicable effect.
\end{abstract}

\section{Introduction}

Parallel manipulator is becoming increasingly popular in a large number of applications. It is a complex system with characteristics of multidegree of freedom, multi-input multioutput, high nonlinearity and strong coupling, and so forth. Control of parallel manipulator is very complex and researches mostly focus on kinematic and dynamic control. Dynamic control can meet the demands of high performance more than kinematic control. Dynamic feed forward control and computed torque control are common dynamic control methods [1-3]. Müller and Hufnagel [4] proposed the adaptive and singularity-free inverse dynamics models for control of parallel manipulators, Achim and Matthias [5] used the dynamics model as a feed forward on a handheld parallel mechanism to reduce the disturbance, and Farhadmanesh and Rastin [6] designed a new fuzzy model-based controller for complex dynamical systems with application to a 3-RRR spherical parallel manipulator.

Classic PID controller is widely used in various kinds of industrial fields for its simple structure and strong robustness. However, classic PID controller cannot meet the different performance requirements simultaneously. 2-DOF control and Internal Model Control are introduced to improve the performance of parallel manipulator [7-10].

First, the servo control strategy of 6PUS-UPU parallel manipulator is analyzed. The PID control is combined with 2-DOF and Internal Model Control to redesign servo control loops of the parallel manipulator. Next the hybrid force/position control of parallel manipulator is proposed. Then the entire model of the 6PUS-UPU parallel manipulator is established, and the mechatronic cosimulation is carried out. Finally the control method is verified by actual prototype experiment.

\section{Control Strategy of 6PUS-UPU Parallel Manipulator}

On the basis of the original current loop and velocity loop, the position loop is redesigned to improve control precision and stability of the parallel manipulator.

2.1. Overview of 6PUS-UPU Parallel Manipulator. A novel 6PUS/UPU parallel manipulator is shown in Figure 1, including the fixed platform, the moving platform, and connected 


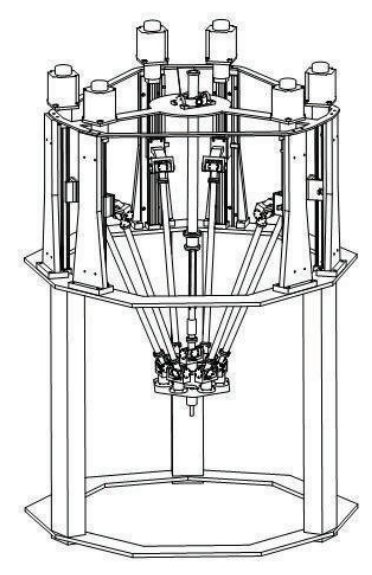

FIGURE 1: 6PUS-UPU parallel manipulator.

branches. The fixed platform and the moving platform are connected with six PUS (prismatic pair-universal pair-sphere pair) actuated branches and a passive UPU (universal jointprismatic pair-universal joint) middle branch. Because one degree of freedom is constrained by the UPU branch, the moving platform only can achieve five degrees of freedom. The parallel manipulator is driven redundantly for its six actuated branches.

2.2. 2-DOF PID Control Based on IMC. For modern control systems, it is hoped to have good position tracking characteristics and good antidisturbance capacity simultaneously. Traditional control methods such as PID control belong to one degree of freedom control and only have one adjustable parameter. The control systems with one adjustable parameter are difficult to meet the requirement of good position tracking characteristics and good antidisturbance capacity. 2-DOF control is designed to solve the problem and two separate parameters are adjusted to have the two abovementioned performances.

The servo control system of 6PUS-UPU parallel manipulator is composed of position loop, velocity loop, and current loop.

(1) Current loop is the innermost loop in the servo system. Current loop consists of the current controller, SPWM, filters, and other components. Proportional and integral controls are used in current controller to have the fastest response among the three loops. The transfer function of current controller is expressed as

$$
G_{i}(s)=\frac{K_{i}\left(T_{i} s+1\right)}{T_{i} s},
$$

where $K_{i}$ is the proportional gain factor and $T_{i}=L / R$ is the integral gain factor.

The control objects of current loop are inverter and armature circuit. The inverter is a conversion circuit from DC to AC. In PMSM control system, SPWM voltage inverter is used and considered as a first-order damp element. The transfer function of inverter is expressed as

$$
G_{\text {ivt }}(s)=\frac{K_{n}}{T_{n} s+1},
$$

where $K_{n}$ is control gain factor and $T_{n}$ is time constant.

The armature circuit is considered as $R-L$ model and its transfer function is expressed as

$$
G_{r l}(s)=\frac{1}{R+L s},
$$

where $R$ is armature resistance and $L$ is armature inductance.

In order to remove the noisy signal, a feedback filter is designed as a first-order damp element. The transfer function of the feedback filter is expressed as

$$
G_{c f}(s)=\frac{K_{c f}}{T_{c f} s+1},
$$

where $K_{c f}$ is gain factor and $T_{c f}$ is time constant.

The signal filter is designed to offset the delay caused by the feedback signal. Its transfer function is a first-order damp element and the transfer function is expressed as

$$
G_{x i}(s)=\frac{1}{T_{c f} s+1} .
$$

In current loop, $I_{r}$ is input current and $I_{m}$ is output current.

(2) Velocity loop is used to improve the antidisturbance capacity by reducing error between output velocity and given velocity. PI control is used and its transfer function is expressed as

$$
G_{v}(s)=\frac{K_{v}\left(T_{v} s+1\right)}{T_{v} s},
$$

where $K_{v}$ is proportional gain factor and $T_{v}$ is integral gain factor.

Feedback filter is also needed in velocity loop and its transfer function is expressed as

$$
G_{w}(s)=\frac{K_{w}}{T_{w} s+1},
$$

where $K_{w}$ is gain factor and $T_{w}$ is time constant.

The signal filter is also added to offset the delay and its transfer function is expressed as

$$
G_{x v}(s)=\frac{1}{T_{w} s+1} .
$$

In velocity loop, $K_{t}$ is torque constant of the motor, $J$ is inertia of the motor, $T_{e}$ is torque of the motor, $T_{L}$ is torque of the load, $\omega_{r}$ is input angular velocity, and $\omega_{m}$ is output angular velocity.

(3) Position loop is the outermost loop and is redesigned based on current loop and velocity loop. The block diagram of three servo loops is shown in Figure 2. In position loop, $\theta^{\prime}$ is 


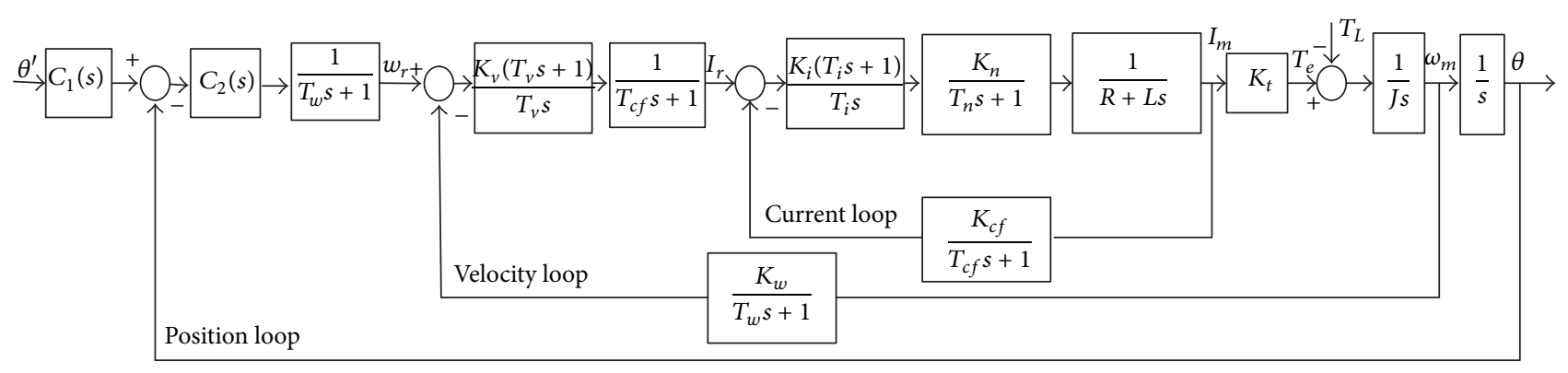

FIGURE 2: Block diagram of three control loops.

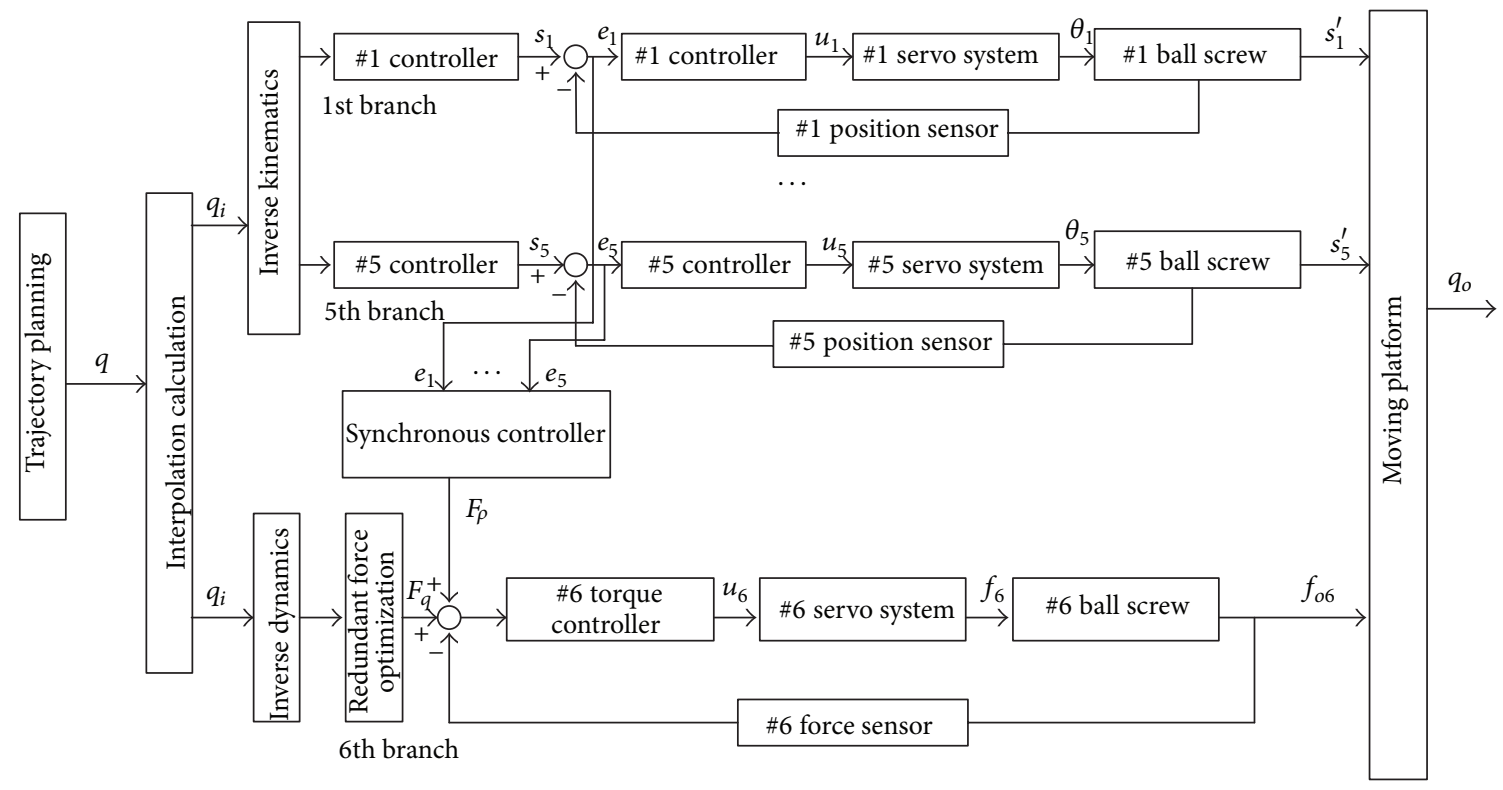

FIGURE 3: Force/position hybrid control.

input angle and $\theta$ is output angle; $C_{1}(s)$ and $C_{2}(s)$ are 2-DOF IMC controllers, respectively.

The mathematical models of $C_{1}(s)$ and $C_{2}(s)$ are expressed as

$$
\begin{aligned}
& C_{1}(s)=\frac{3 \lambda_{1} s+1}{3 \lambda_{2} s+1}, \\
& C_{2}(s)=\frac{\left(3 \lambda_{2} s+1\right)\left(T_{0} s+1\right)}{3 \lambda_{2}^{2} s\left(\left(\lambda_{2} / 3\right) s+1\right)},
\end{aligned}
$$

where $\lambda_{1}, \lambda_{2}$ are control parameters and $T_{0}$ is time constant.

2.3. Force/Position Hybrid Control Strategy. The force/position hybrid control of 6PUS-UPU parallel manipulator is proposed as shown in Figure 3. For five nonredundant branches, position control modes are used, and for the redundant branch force control mode is used.

The cross-coupling relationship between driving axes should be taken into consideration. The coupling position errors of five nonredundant branches from the 1st to the 5th branch in joint space are fed back to the 6 th redundant driven branch. The coupling position errors are converted to the force signal to make compensation in synchronous controller. So the position precision and torque balance of the parallel manipulator will be improved simultaneously.

It is assumed that the theoretical position of branch $i$ is $s_{i}(t)$ and the actual position is $s_{i}^{\prime}(t)$ at the time $t$. Then, the position error of the branch at the time $t$ is as follows:

$$
e_{i}(t)=s_{i}(t)-s_{i}^{\prime}(t) \quad i=1 \sim 5 .
$$

Only when the error of each branch is close to zero would the synchronous control be realized. Its variables $\xi_{i}(t)(i=1 \sim$ 10) are defined as

$$
\xi_{i}(t)=e_{n}(t)-e_{m}(t) \quad n=1 \sim 4, m=(n+1) \sim 5 .
$$

According to the minimum norm theory, the error of synchronous control is defined as

$$
\rho(t)=\int_{0}^{t} \sqrt{\frac{1}{10} \sum_{i=1}^{10} \xi_{i}(w)} \mathrm{d} w .
$$

The driving force $F_{6}$ can be divided into two parts for the redundant driven branch. One is the driving force from 


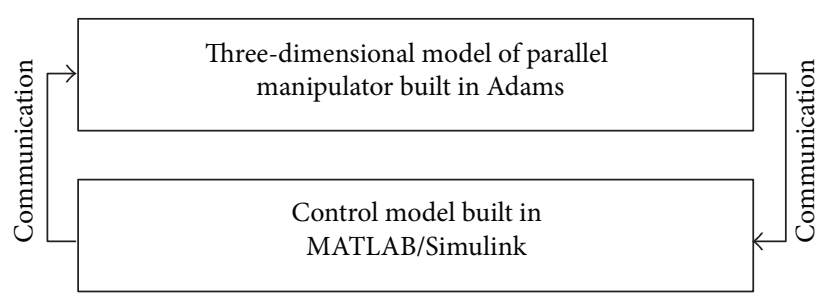

FIgURE 4: Structure of Adams and MATLAB cosimulation.

the optimized inverse redundant torque $F_{q}$ and the other is the driving force from the compensation of synchronous control error $F_{q}$. The total driving force is defined as

$$
F_{6}=F_{q}+F_{\rho} .
$$

By means of computing torque method, the relationship between the redundant driving force and the coupling errors can be expressed as

$$
F_{\rho}=m_{6} \rho^{\prime \prime}(t)+c_{6} \rho^{\prime}(t)+g \rho(t),
$$

where $m_{6}, c_{6}$, and $g$ are mass, coefficient of Coriolis force, and gravitational acceleration of the 6th branch; $\rho^{\prime}(t)$ and $\rho^{\prime \prime}(t)$ are velocity error and acceleration error of synchronous control.

Eventually, the hybrid control with position and force is realized effectively.

\section{Mechatronic Cosimulation}

3.1. Cosimulation Principle. The 3D model of the parallel manipulator is established in Adams and the control model of the parallel manipulator is established in Simulink module of MATLAB. The mechanical model is combined with the control system of the parallel manipulator to realize mechatronic cosimulation.

The cosimulation diagram between Adams and MATLAB is shown in Figure 4.

The major step is shown in Figure 5.

3.2. Examples of Cosimulation. As shown in Figure 6, 3D model of the parallel manipulator is established in Adams. MOTION_1 to MOTION_5 are added as position driver of five nonredundant branches and FORCE is added as force driver of the redundant branch.

The control models of nonredundant branches and the redundant branch are established in Simulink module of MATLAB. Position control is adopted in five nonredundant branches and force control is used in the redundant branch. The parameters in relevant transfer function mentioned above are listed in Table 1.

The given trajectory is a major arc of $200 \mathrm{~mm}$ radius in cosimulation. The output response of the moving platform is shown in Figure 7.

In Figure 7, the output of the moving platform with 2DOF IMC is closer to the theoretical value than the traditional control and the accuracy of tracking position is improved effectively.
TABle 1: Parameters list.

\begin{tabular}{lc}
\hline Parameter & Data \\
\hline$\lambda_{1}$ & 0.018 \\
$\lambda_{2}$ & 0.15 \\
$T_{0}$ & $0.01475 \mathrm{~s}$ \\
$J$ & $0.000188 \mathrm{~kg} \cdot \mathrm{m}^{2}$ \\
$K_{v}$ & 0.015 \\
$T_{v}$ & $5 \mathrm{~s}$ \\
$K_{w}$ & 1 \\
$T_{w}$ & $0.01 \mathrm{~s}$ \\
$K_{i}$ & 1 \\
$T_{i}$ & $0.00208 \mathrm{~s}$ \\
$K_{\mathrm{cf}}$ & 1 \\
$T_{\mathrm{cf}}$ & $0.00016 \mathrm{~s}$ \\
$K_{n}$ & 4.43 \\
$T_{n}$ & $0.0001 \mathrm{~s}$ \\
$R$ & $1.3 \Omega$ \\
$L$ & $0.0027 \mathrm{H}$ \\
\hline
\end{tabular}

\section{Experiment and Application}

The self-developed prototype of 6PUS-UPU parallel manipulator is shown in Figure 8.

In the experiment, the trajectory is from the starting point $(0,0,0)$, through the point $(-100,-100,0)$, to the final point $(100,100,0)$.

The two experiments were done in PID control mode and hybrid synchronous control mode, respectively. The actual trajectories of the moving platform are measured by the laser interferometer. By comparing the data, the errors of the hybrid control can be reduced by about $15 \%$ compared to PID control mode.

As shown in Table 2, the output torque peak in two control modes of five nonredundent branches is recorded by oscilloscope. The torque peak of the hybrid synchronous control can be reduced by about $10 \%$ compared with PID control mode and the output torques are banlanced.

Based on this, two types of actual compliance operation are realized in 6PUS-UPU parallel manipulator. As shown in Figure 9, peg in hole (a) and surface tracking (b) both have good effects.

\section{Conclusion}

The control method combining 2-DOF control and Internal Model Control was optimized. On the basis of current loop 
TABLE 2: Output torque peak table (rounded, unit: $\mathrm{Nm}$ ).

\begin{tabular}{lccccc}
\hline Control mode & Branch one & Branch two & Branch three & Branch four & Branch five \\
\hline PID control & 485 & 2830 & 3891 & 3590 & 1381 \\
Synchronous control & 440 & 2516 & 3463 & 3265 & 1207 \\
\hline
\end{tabular}

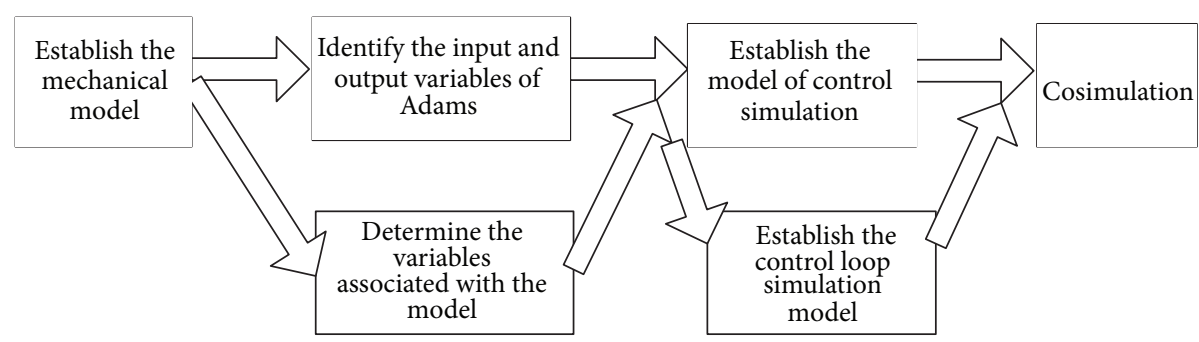

FIGURE 5: Flowchart of cosimulation.

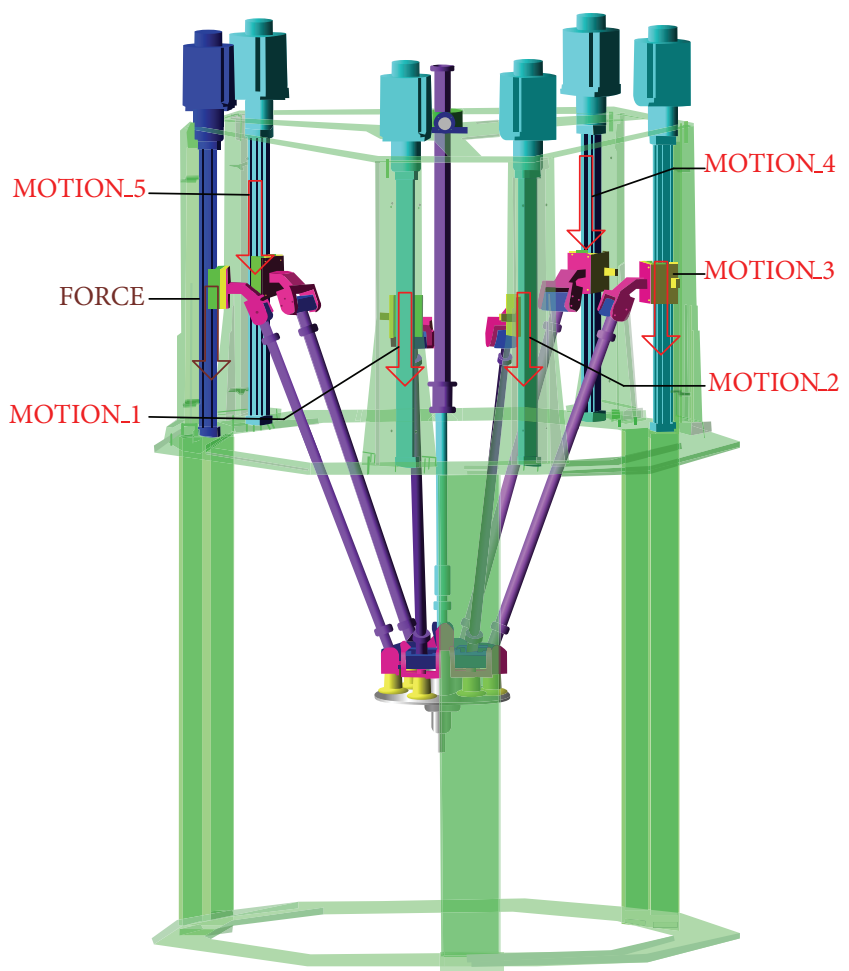

Figure 6: 3D model in Adams.

and velocity loop, position loop was redesigned to improve stability and position precision simultaneously. Based on cross-coupling, the force/position hybrid control of parallel manipulator is realized. Mechatronic cosimulation and the experiment of 6PUS-UPU parallel manipulator were carried out and both high stability and good position precision are verified. It is effective for peg in hole and surface tracking by actual operation and it should be also applicable to glass cleaning, egg grasping, component assembly, space docking, and so forth.

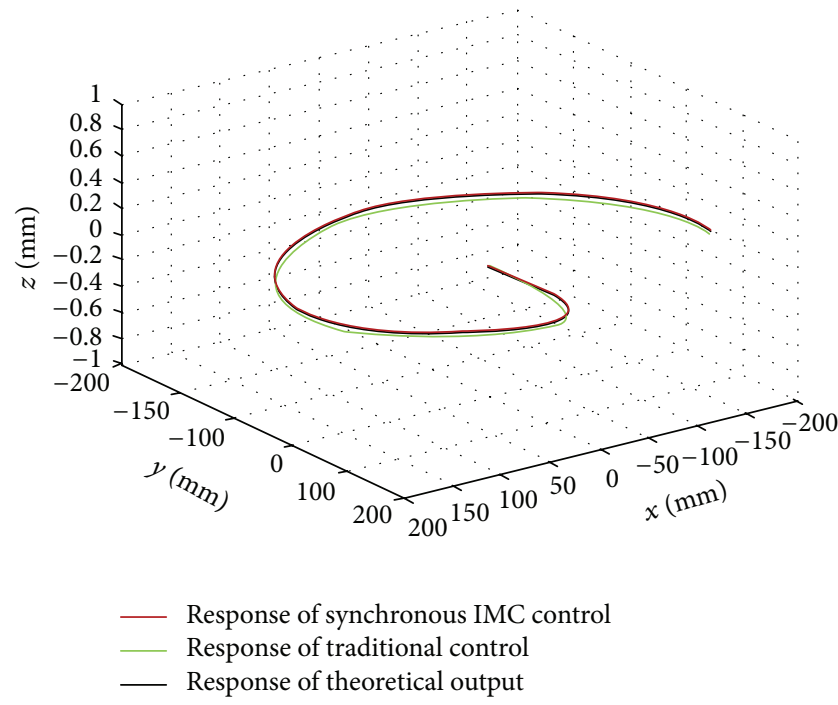

FIGURE 7: 3D comparison chart of output response.

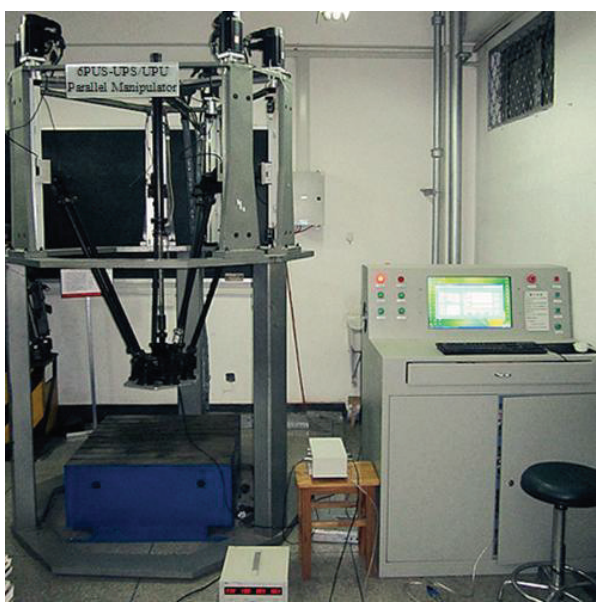

FIGURE 8: The prototype of 6PUS-UPU parallel manipulator. 


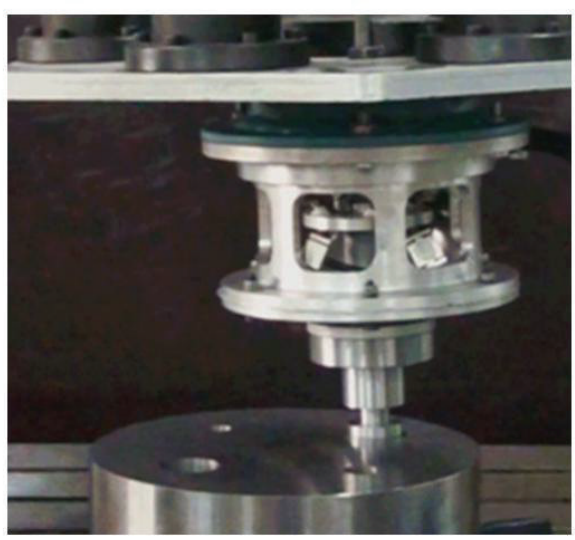

(a) Peg in hole

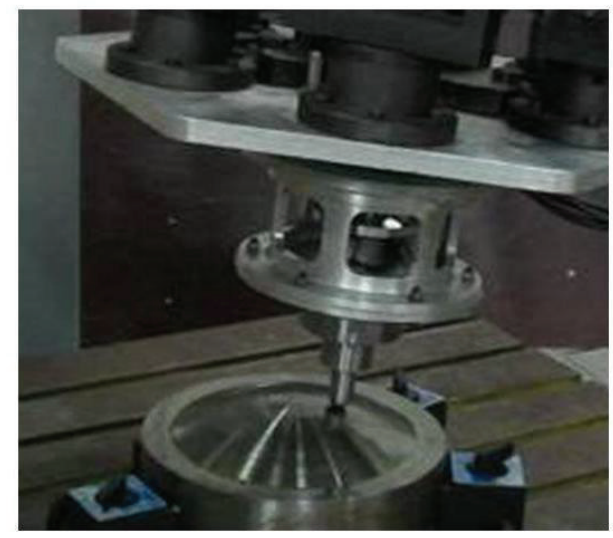

(b) Surface tracking

FIGURE 9: Application of 6PUS-UPU parallel manipulator.

\section{Conflict of Interests}

The authors declare that there is no conflict of interests regarding the publication of this paper.

\section{Acknowledgments}

This study was supported by the Natural Science Fund, China (51275439), and Natural Science Fund of Hebei Province, China (E2012203130).

\section{References}

[1] S. A. Ajwad, R. U. Islam, U. Iqbal, and J. Iqbal, "Modeling and computed torque control of a 6 degree of freedom robotic arm," in Proceedings of the IEEE International Conference on Robotics and Emerging Allied Technologies in Engineering, pp. 133-138, 2014.

[2] G. Zhang, J. H. Wu, P. K. Liu, and H. Ding, "Dynamic analysis and model-based feedforward control of a 2-dof translational parallel manipulator driven by linear motors," Industrial Robot, vol. 40, no. 6, pp. 597-609, 2013.

[3] Z. Y. Yang, J. Y. Wu, and J. P. Mei, "Motor-mechanism dynamic model based neural network optimized computed torque control of a high speed parallel manipulator," Mechatronics, vol. 17, no. 7, pp. 381-390, 2007.

[4] A. Müller and T. Hufnagel, "Adaptive and singularity-free inverse dynamics models for control of parallel manipulators with actuation redundancy," in Proceedings of the ASME Design Engineering Technical Conference, vol. 6, pp. 989-997, August 2011.

[5] W. Achim and N. Matthias, "Disturbance feed forward control of a handheld parallel robot," in Proceedings of the International Conference on Informatics in Control, Automation and Robotics, vol. 1, pp. 44-51, 2007.

[6] M. Farhadmanesh, M. A. Rastin, S. A. A. Moosavian, and M. Taherynezhad, "Design of a new fuzzy model-based controller for complex dynamical systems with application to a 3-RRR spherical parallel manipulator," in Proceedings of the Iranian Conference on Fuzzy Systems, pp. 1-5, 2013.
[7] F. Jin and L. Dong, "Research on PMSM servo system based on internal module and robust 2-DOF control structure," in Proceedings of the Chinese Control and Decision Conference, pp. 5393-5396, 2009.

[8] S. Alcántara, C. Pedret, R. Vilanova, and S. Skogestad, "Generalized internal model control for balancing input/output disturbance response," Industrial and Engineering Chemistry Research, vol. 50, no. 19, pp. 11170-11180, 2011.

[9] A. M. Y. Luk, E. H. K. Fung, and W. C. Gan, “2-DOF planar motion control system using model reference adaptive control algorithm," in Proceedings of ASME International Mechanical Engineering Congress and Exposition (IMECE '12), pp. 271-280, Houston, Tex, USA, November 2012.

[10] W. W. Shang and S. Cong, "Robust nonlinear control of a planar 2-DOF parallel manipulator with redundant actuation," Robotics and Computer-Integrated Manufacturing, vol. 30, no. 6, pp. 597-604, 2014. 

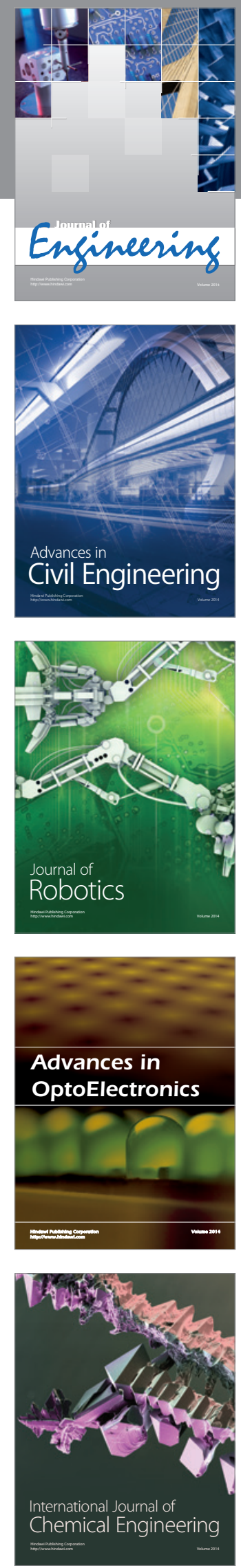

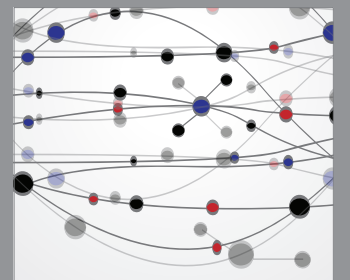

The Scientific World Journal
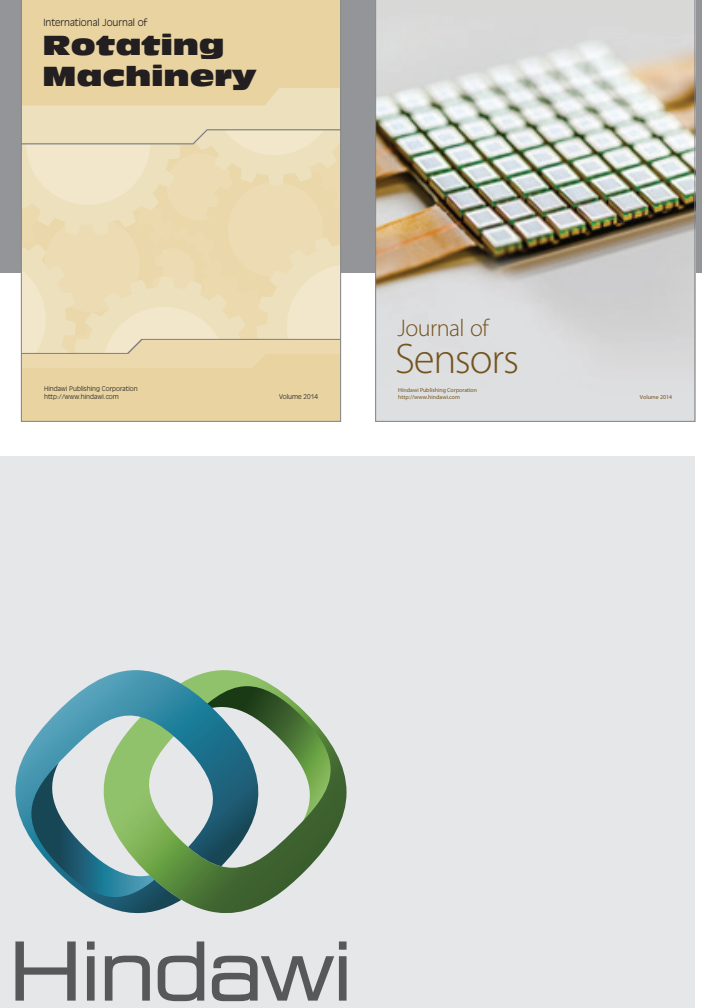

Submit your manuscripts at http://www.hindawi.com
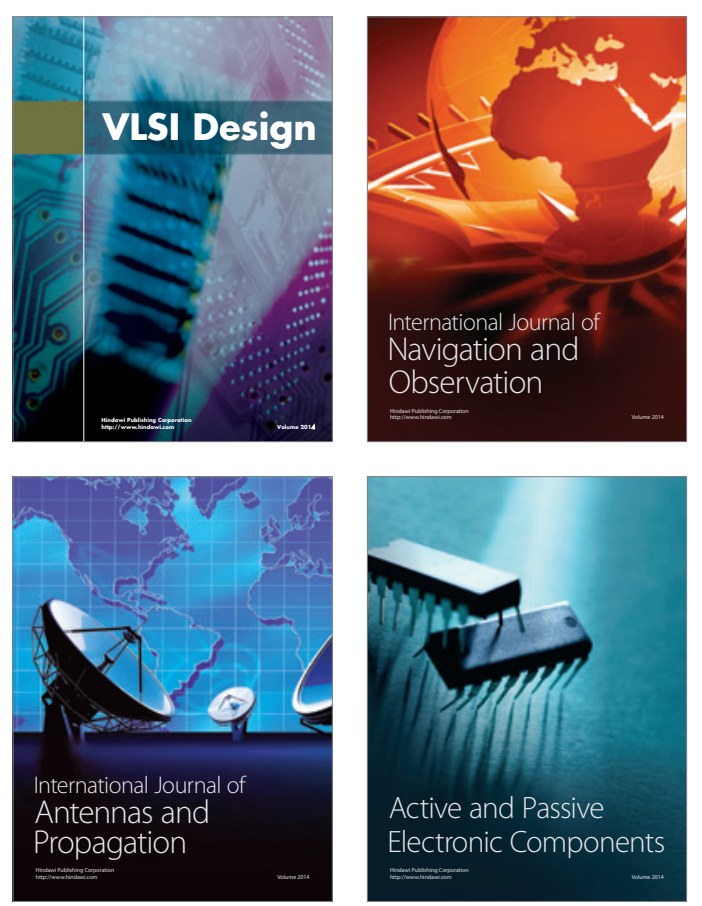
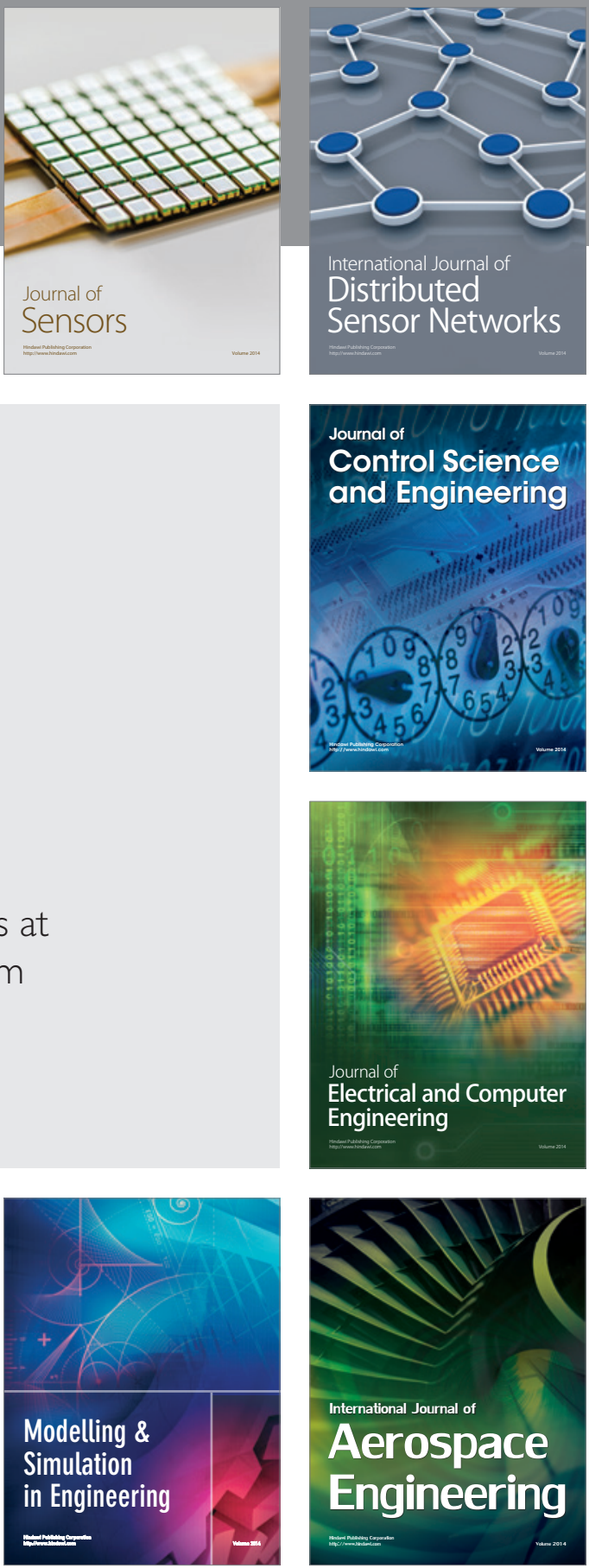

Journal of

Control Science

and Engineering
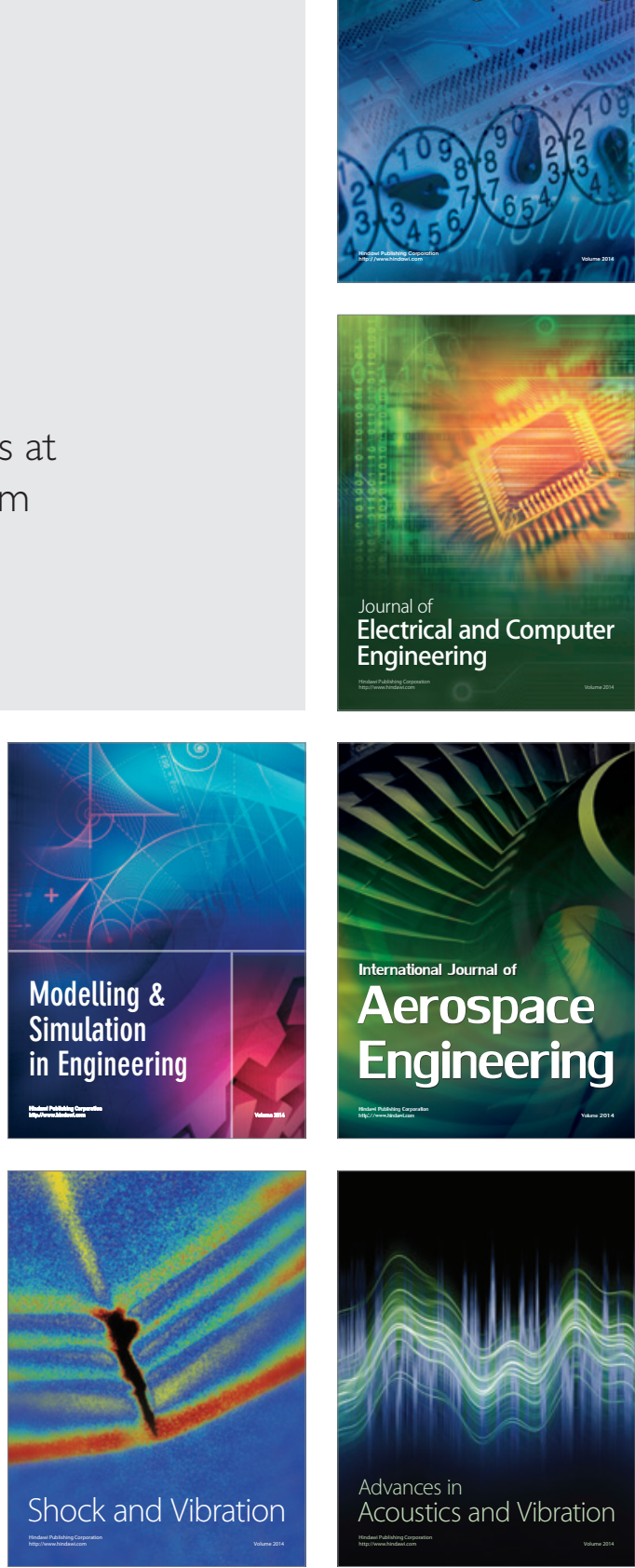